九州大学学術情報リポジトリ

Kyushu University Institutional Repository

\title{
THE TRANSITIVE CLOSURE OF FUZZY RELATIONS WITH A CONTRACTION PROPERTY
}

Yoshida, Yuj i

Faculty of Economics and Business Administration, Kitakyushu University

https://doi.org/10.5109/13446

出版情報: Bulletin of informatics and cybernetics. 27 (1)，pp.121-128，1995-03. Research Association of Statistical Sciences

バージョン:

権利関係 : 


\title{
THE TRANSITIVE CLOSURE OF FUZZY RELATIONS WITH A CONTRACTION PROPERTY
}

\author{
By
}

\section{Yuji YoshidA $^{*}$}

\begin{abstract}
This paper analyses transitive closures of fuzzy relations on a compact metric space with a contraction property in Kurano et al. (1992). We show that the transitive closure is a unique solution of a fuzzy relational equation and also has the same contraction property.
\end{abstract}

\section{Introduction and notations}

Let $E$ be a compact metric space and $d$ be a metric on $E . \mathcal{C}(E)$ denotes the collection of all non-empty closed subsets of $E$. We put $d(x, D):=\inf _{y \in D} d(x, y), x \in$ $E, D \in \mathcal{C}(E)$. Let $\rho$ be the Hausdorff metric on $\mathcal{C}(E)$. Then it is well-known ([1]) that $(\mathcal{C}(E), \rho)$ is a compact metric space. Let $\mathcal{F}(E)$ be the set of all fuzzy sets $\tilde{s}: E \mapsto[0,1]$ which are upper semi-continuous and satisfy $\sup _{x \in E} \tilde{s}(x)=1$.

For a fuzzy relation $\tilde{r}: E \times E \mapsto[0,1]$ satisfying $\tilde{r}(x, \cdot) \in \mathcal{F}(E)(x \in E)$, we define a map $\tilde{r}_{\alpha}: \mathcal{C}(E) \mapsto \mathcal{C}(E)(\alpha \in[0,1])$ by

$$
\tilde{r}_{\alpha}(D):= \begin{cases}\{y \mid \tilde{r}(x, y) \geq \alpha \text { for some } x \in D\} & \text { for } \alpha \neq 0, D \in \mathcal{C}(E) \\ \operatorname{cl}\{y \mid \tilde{r}(x, y)>0 \text { for some } x \in D\} & \text { for } \alpha=0, D \in \mathcal{C}(E),\end{cases}
$$

where cl denotes the closure of a set. Let $\mathcal{R}(E)$ be the set of all fuzzy relations $\tilde{r}$ : $E \times E \mapsto[0,1]$ which satisfy $\tilde{r}(x, \cdot) \in \mathcal{F}(E)(x \in E)$ and

$$
\sup _{\alpha \in[0,1]} \rho\left(\tilde{r}_{\alpha}(y), \tilde{r}_{\alpha}(x)\right) \rightarrow 0(y \rightarrow x) \quad \text { for } x \in E .
$$

We denote the maximum operation and the minimum operation by $\vee$ and $\wedge$, respectively. Let $\tilde{q} \in \mathcal{R}(E)$ be a continuous fuzzy relation. We define sequences of fuzzy relations $\left\{\tilde{q}^{n}\right\}_{n=1}^{\infty}$ and $\left\{\tilde{c}^{m}\right\}_{m=1}^{\infty}$ by

$$
\begin{gathered}
\tilde{q}^{1}:=\tilde{q} \quad \text { and } \quad \tilde{q}^{n+1}(x, y):=\sup _{z \in E}\left\{\tilde{q}(x, z) \wedge \tilde{q}^{n}(z, y)\right\}, \quad x, y \in E, n=1,2, \cdots ; \\
\tilde{c}^{m}(x, y):=\bigvee_{n=1,2, \cdots, m} \tilde{q}^{n}(x, y), \quad x, y \in E, m=1,2, \cdots
\end{gathered}
$$

\footnotetext{
* Faculty of Economics and Business Administration, Kitakyushu University, 4-2-1 Kitagata, Kokuraminami, Kitakyushu 802, Japan.
} 
If $E$ is finite, then the transitive closure of the fuzzy relation $\tilde{q}$ is given ([5]) by

$$
\bigvee_{n=1,2, \cdots} \tilde{q}^{n}(x, y), \quad x, y \in E
$$

This paper discusses the transitive closure when $E$ is a general compact metric space. From now on we assume the following contraction property.

Assumption (Contraction property, [2]). There exists a real number $\beta(0<\beta<1)$ satisfying the following condition :

$$
\rho\left(\tilde{q}_{\alpha}(A), \tilde{q}_{\alpha}(B)\right) \leq \beta \rho(A, B) \quad \text { for all } A, B \in \mathcal{C}(E) \text { and all } \alpha \in[0,1] .
$$

We call $\beta$ a contraction factor.

Lemma 1. The condition (1.6) is equivalent to the following condition :

$$
\rho\left(\tilde{q}_{\alpha}(x), \tilde{q}_{\alpha}(y)\right) \leq \beta d(x, y) \quad \text { for all } x, y \in E \text { and all } \alpha \in[0,1],
$$

where $\tilde{q}_{\alpha}(x):=\tilde{q}_{\alpha}(\{x\})(x \in E, \alpha \in[0,1])$.

Proof. We can obtain (1.7) from (1.6), taking $A=\{x\}, B=\{y\}$. Conversely we assume (1.7). Let $A, B \in \mathcal{C}(E)$. Since $\tilde{q}_{\alpha}(x) \subset \tilde{q}_{\alpha}(A)$ for all $x \in A$ and $\tilde{q}_{\alpha}(y) \subset \tilde{q}_{\alpha}(B)$ for all $y \in B$,

$$
\begin{aligned}
\rho\left(\tilde{q}_{\alpha}(A), \tilde{q}_{\alpha}(B)\right) & =\max \left\{\max _{x^{\prime} \in \tilde{q}_{\alpha}(A)} d\left(x^{\prime}, \tilde{q}_{\alpha}(B)\right), \max _{y^{\prime} \in \tilde{q}_{\alpha}(B)} d\left(\tilde{q}_{\alpha}(A), y^{\prime}\right)\right\} \\
& \leq \max \left\{\max _{x^{\prime} \in \tilde{q}_{\alpha}(A)} \min _{y \in B} d\left(x^{\prime}, \tilde{q}_{\alpha}(y)\right), \max _{y^{\prime} \in \tilde{q}_{\alpha}(B)} \min _{x \in A} d\left(\tilde{q}_{\alpha}(x), y^{\prime}\right)\right\}
\end{aligned}
$$

Here since $\tilde{q}_{\alpha}(A)=\bigcup_{x \in A} \tilde{q}_{\alpha}(x)$, we have

$$
\max _{x^{\prime} \in \bar{q}_{\alpha}(A)} \min _{y \in B} d\left(x^{\prime}, \tilde{q}_{\alpha}(y)\right)=\max _{x \in A} \max _{x^{\prime} \in \tilde{q}_{\alpha}(x)} \min _{y \in B} d\left(x^{\prime}, \tilde{q}_{\alpha}(y)\right) \leq \max _{x \in A} \min _{y \in B} \rho\left(\tilde{q}_{\alpha}(x), \tilde{q}_{\alpha}(y)\right) .
$$

Similarly

$$
\max _{y^{\prime} \in \tilde{q}_{\alpha}(B)} \min _{x \in A} d\left(\tilde{q}_{\alpha}(x), y^{\prime}\right) \leq \max _{y \in B} \min _{x \in A} \rho\left(\tilde{q}_{\alpha}(x), \tilde{q}_{\alpha}(y)\right)
$$

Therefore we obtain

$$
\rho\left(\tilde{q}_{\alpha}(A), \tilde{q}_{\alpha}(B)\right) \leq \max \left\{\max _{x \in A} \min _{y \in B} \rho\left(\tilde{q}_{\alpha}(x), \tilde{q}_{\alpha}(y)\right), \max _{y \in B} \min _{x \in A} \rho\left(\tilde{q}_{\alpha}(x), \tilde{q}_{\alpha}(y)\right)\right\} .
$$

From (1.7), we get

$$
\rho\left(\tilde{q}_{\alpha}(A), \tilde{q}_{\alpha}(B)\right) \leq \beta \max \left\{\max _{x \in A} \min _{y \in B} d(x, y), \max _{y \in B} \min _{x \in A} d(x, y)\right\}=\beta \rho(A, B) .
$$

Therefore we obtain (1.6).

Definition 1 ([3]). For $\tilde{r}_{n}, \tilde{r} \in \mathcal{R}(E)$,

$$
\lim _{n \rightarrow \infty} \tilde{r}_{n}=\tilde{r}
$$


means

$$
\sup _{\alpha \in[0,1]} \rho\left(\tilde{r}_{n, \alpha}(D), \tilde{r}_{\alpha}(D)\right) \rightarrow 0 \quad(n \rightarrow \infty) \quad \text { for } D \in \mathcal{C}(E),
$$

where $\tilde{r}_{n, \alpha}, \tilde{r}_{\alpha}$ are defined by (1.1) for the fuzzy relations $\tilde{r}_{n}, \tilde{r}$, respectively.

LEMma 2 ([2,Lemma 2]). Suppose that a family of subsets $\left\{D_{\alpha} \mid \alpha \in[0,1]\right\} \subset \mathcal{C}(E)$ satisfies the following conditions:

(i) $D_{\alpha} \subset D_{\alpha^{\prime}}$ for $\alpha^{\prime} \leq \alpha$.

(ii) $\lim _{\alpha^{\prime} \uparrow \alpha} D_{\alpha^{\prime}}=D_{\alpha}$, i.e., $\lim _{\alpha^{\prime} \uparrow \alpha} \rho\left(D_{\alpha^{\prime}}, D_{\alpha}\right)=0 \quad$ for $\alpha \in(0,1]$.

Then it holds that

$$
\lim _{\alpha^{\prime} \uparrow \alpha} \tilde{q}_{\alpha^{\prime}}\left(D_{\alpha^{\prime}}\right)=\tilde{q}_{\alpha}\left(D_{\alpha}\right) \quad \text { for } \alpha \in(0,1]
$$

Lemma 3. We suppose that a family of subsets $\left\{D_{\alpha}(x) \mid x \in E, \alpha \in[0,1]\right\}(\subset \mathcal{C}(E))$ satisfies the following conditions (i) - (iii) :

(i) $D_{\alpha}(x) \subset D_{\alpha^{\prime}}(x) \quad$ for $x \in E, 0 \leq \alpha^{\prime}<\alpha \leq 1$.

(ii) $\lim _{\alpha^{\prime} \uparrow \alpha} D_{\alpha^{\prime}}(x)=D_{\alpha}(x)$ for $x \in E, \alpha \in(0,1]$.

(iii) $\sup _{\alpha \in[0,1]} \rho\left(D_{\alpha}(y), D_{\alpha}(x)\right) \rightarrow 0 \quad(y \rightarrow x) \quad$ for $x \in E$.

Then

$$
\tilde{r}(x, y):=\sup _{\alpha \in[0,1]}\left\{\alpha \wedge I_{D_{\alpha}(x)}(y)\right\}, \quad x, y \in E,
$$

satisfies $\tilde{r} \in \mathcal{R}(E)$ and $\tilde{r}_{\alpha}(x)=D_{\alpha}(x)$ for all $x \in E, \alpha \in[0,1]$.

Proof. Fix any $x \in E$. By [4], from the conditions (i) and (ii), we have $\tilde{r}(x, \cdot) \in$ $\mathcal{F}(E)$ and $\tilde{r}_{\alpha}(x)=\{y \in E \mid \tilde{r}(x, y) \geq \alpha\}=D_{\alpha}(x)$ for $\alpha \in(0,1]$. Therefore (1.2) holds from (iii). Thus we get $\tilde{r} \in \mathcal{R}(E)$.

We define maps $\tilde{q}_{\alpha}^{n}: C(E) \mapsto C(E)(n=1,2, \cdots, \alpha \in[0,1])$ by $\tilde{q}_{\alpha}^{1}:=\tilde{q}_{\alpha}$ and $\tilde{q}_{\alpha}^{n+1}:=\tilde{q}_{\alpha}\left(\tilde{q}_{\alpha}^{n}\right)(n=1,2, \cdots)$.

Lemma 4. Let $\alpha \in[0,1]$. Then :

(i) $\left(\tilde{q}^{n}\right)_{\alpha}(D)=\tilde{q}_{\alpha}^{n}(D), \quad D \in \mathcal{C}(E)$ for $n=1,2, \cdots$;

(ii) $\left(\tilde{c}^{m}\right)_{\alpha}(D)=\bigcup_{n=1,2, \cdots, m} \tilde{q}_{\alpha}^{n}(D), \quad D \in \mathcal{C}(E) \quad$ for $m=1,2, \cdots$.

Proof. We have (i) from [2,Lemma 1] and (1.3). Further (ii) is trivial from (1.4).

LEMMA 5. It holds that

$$
\rho(A \cup C, B \cup D) \leq \max \{\rho(A, B), \rho(C, D)\} \quad \text { for } A, B, C, D \in \mathcal{C}(E)
$$


Proof. Let $A, B, C, D \in \mathcal{C}(E)$. Then

$$
\begin{aligned}
\rho( & (A \cup C, B \cup D) \\
& =\max \left\{\max _{x \in A \cup C} d(x, B \cup D), \max _{y \in B \cup D} d(A \cup C, y)\right\} \\
& =\max \left\{\max _{x \in A} d(x, B \cup D), \max _{y \in B} d(A \cup C, y), \max _{x \in C} d(x, B \cup D), \max _{y \in D} d(A \cup C, y)\right\} \\
& \leq \max \left\{\max _{x \in A} d(x, B), \max _{y \in B} d(A, y), \max _{x \in C} d(x, D), \max _{y \in D} d(C, y)\right\} \\
& =\max \{\rho(A, B), \rho(C, D)\} .
\end{aligned}
$$

Therefore we obtain this lemma.

\section{Main results}

We discuss the convergence of the sequence of fuzzy relations $\left\{\tilde{c}^{m}\right\}_{m=1}^{\infty}$.

Theorem 1.

(i) There exists a unique solution $\tilde{c} \in \mathcal{R}(E)$ of the following fuzzy relational equation:

$$
\tilde{c}(x, y)=\tilde{q}(x, y) \vee \max _{z \in E}\{\tilde{c}(x, z) \wedge \tilde{q}(z, y)\} \quad x, y \in E .
$$

(ii) The fuzzy relation $\tilde{c}$ also has the contraction property with the same contraction factor $\beta$.

(iii) The fuzzy relation $\tilde{c}$ equals to the limit of $\left\{\tilde{c}^{m}\right\}_{m=1}^{\infty}$ :

$$
\tilde{c}=\lim _{m \rightarrow \infty} \tilde{c}^{m} \text {. }
$$

Proof. Define a map $T_{x, \alpha}: \mathcal{C}(E) \rightarrow \mathcal{C}(E)(x \in E, \alpha \in[0,1])$ by

$$
T_{x, \alpha}(D):=\tilde{q}_{\alpha}(x) \cup \tilde{q}_{\alpha}(D), \quad D \in \mathcal{C}(E) .
$$

From Lemmas 1 and 5,

$$
\begin{aligned}
\rho\left(T_{x, \alpha}(D), T_{x, \alpha}\left(D^{\prime}\right)\right) & =\rho\left(\tilde{q}_{\alpha}(x) \cup \tilde{q}_{\alpha}(D), \tilde{q}_{\alpha}(x) \cup \tilde{q}_{\alpha}\left(D^{\prime}\right)\right) \\
& \leq \rho\left(\tilde{q}_{\alpha}(D), \tilde{q}_{\alpha}\left(D^{\prime}\right)\right) \\
& \leq \beta \rho\left(D, D^{\prime}\right), \quad D, D^{\prime} \in \mathcal{C}(E), x \in E, \alpha \in[0,1] .
\end{aligned}
$$

Since the metric space $(\mathcal{C}(E), \rho)$ is compact, from the Banach's fixed point theorem, there exists a family $\left\{A_{\alpha}(x) \mid x \in E, \alpha \in[0,1]\right\} \subset \mathcal{C}(E)$ such that

$$
\tilde{q}_{\alpha}(x) \cup \tilde{q}_{\alpha}\left(A_{\alpha}(x)\right)=T_{x, \alpha}\left(A_{\alpha}(x)\right)=A_{\alpha}(x), \quad x \in E, \alpha \in[0,1],
$$

and $\lim _{n \rightarrow \infty} T_{x, \alpha}^{n}(D)=A_{\alpha}(x)$ for any $D \in \mathcal{C}(E)$. From the definition of $\tilde{q}_{\alpha}, T_{x, \alpha^{\prime}}(D)=$ $\tilde{q}_{\alpha^{\prime}}(x) \cup \tilde{q}_{\alpha^{\prime}}(D) \supset \tilde{q}_{\alpha}(x) \cup \tilde{q}_{\alpha}(D)=T_{x, \alpha}(D)$ for $\alpha^{\prime} \leq \alpha$. Inductively we have $T_{x, \alpha^{\prime}}^{n}(D) \supset$ $T_{x, \alpha}^{n}(D)$ for $n=1,2, \cdots$. By letting $n \rightarrow \infty$, we obtain

$$
A_{\alpha^{\prime}}(x) \supset A_{\alpha}(x) \text { for } \alpha^{\prime} \leq \alpha .
$$


Let $\alpha^{\prime} \leq \alpha$. Inductively we have

$$
\begin{aligned}
\rho\left(A_{\alpha}(x), A_{\alpha^{\prime}}(x)\right) & =\rho\left(T_{x, \alpha}^{n}\left(A_{\alpha}(x)\right), T_{x, \alpha^{\prime}}^{n}\left(A_{\alpha^{\prime}}(x)\right)\right) \\
& \leq \rho\left(T_{x, \alpha^{\prime}}^{n}\left(A_{\alpha}(x)\right), T_{x, \alpha}^{n}\left(A_{\alpha}(x)\right)\right)+\rho\left(T_{x, \alpha^{\prime}}^{n}\left(A_{\alpha^{\prime}}(x)\right), T_{x, \alpha^{\prime}}^{n}\left(A_{\alpha}(x)\right)\right) \\
& \leq \rho\left(T_{x, \alpha^{\prime}}^{n}\left(A_{\alpha}(x)\right), T_{x, \alpha}^{n}\left(A_{\alpha}(x)\right)\right)+\beta^{n} \rho\left(A_{\alpha^{\prime}}(x), A_{\alpha}(x)\right), \quad n=1,2, \cdots .
\end{aligned}
$$

Then $\rho\left(A_{\alpha^{\prime}}(x), A_{\alpha}(x)\right)$ is uniformly bounded since $E$ is compact. We put $\rho\left(A_{\alpha^{\prime}}(x), A_{\alpha}(x)\right) \leq$ $M$ for some $M>0$. Therefore

$$
\rho\left(A_{\alpha}(x), A_{\alpha^{\prime}}(x)\right) \leq \rho\left(T_{x, \alpha^{\prime}}^{n}\left(A_{\alpha}(x)\right), T_{x, \alpha}^{n}\left(A_{\alpha}(x)\right)\right)+\beta^{n} M, \quad n=1,2, \cdots .
$$

By Lemma 2, we have $\lim _{\alpha^{\prime} \uparrow \alpha} T_{x, \alpha^{\prime}}\left(A_{\alpha}(x)\right)=\lim _{\alpha^{\prime} \uparrow \alpha}\left\{\tilde{q}_{\alpha^{\prime}}(x) \cup \tilde{q}_{\alpha^{\prime}}\left(A_{\alpha}(x)\right)\right\}=\tilde{q}_{\alpha}(x) \cup$ $\tilde{q}_{\alpha}\left(A_{\alpha}(x)\right)=T_{x, \alpha}\left(A_{\alpha}(x)\right)$. Repeating these arguments inductively, we have

$$
\lim _{\alpha^{\prime} \uparrow \alpha} T_{x, \alpha^{\prime}}^{n}\left(A_{\alpha}(x)\right)=T_{x, \alpha}^{n}\left(A_{\alpha}(x)\right), \quad n=1,2, \cdots .
$$

Therefore (2.4) implies

$$
\lim _{\alpha^{\prime} \uparrow \alpha} \rho\left(A_{\alpha}(x), A_{\alpha^{\prime}}(x)\right) \leq \beta^{n} M, \quad n=1,2, \cdots
$$

By letting $n \rightarrow \infty$, we obtain

$$
\lim _{\alpha^{\prime} \uparrow \alpha} A_{\alpha^{\prime}}(x)=A_{\alpha}(x)
$$

Let $\alpha \in[0,1]$ and $x, y \in E$. From Lemma 5 and the contraction property of $\tilde{q}$, we have

$$
\begin{aligned}
\rho\left(T_{y, \alpha}(D), T_{x, \alpha}\left(D^{\prime}\right)\right) & =\rho\left(\tilde{q}_{\alpha}(y) \cup \tilde{q}_{\alpha}(D), \tilde{q}_{\alpha}(x) \cup \tilde{q}_{\alpha}\left(D^{\prime}\right)\right) \\
& \leq \max \left\{\rho\left(\tilde{q}_{\alpha}(y), \tilde{q}_{\alpha}(x)\right), \rho\left(\tilde{q}_{\alpha}(D), \tilde{q}_{\alpha}\left(D^{\prime}\right)\right)\right\} \\
& \leq \beta \max \left\{d(y, x), \rho\left(D, D^{\prime}\right)\right\}, \quad D, D^{\prime} \in \mathcal{C}(E)
\end{aligned}
$$

Repeating these arguments inductively, we have

$$
\rho\left(T_{y, \alpha}^{n}(D), T_{x, \alpha}^{n}\left(D^{\prime}\right)\right) \leq \max \left\{\beta d(y, x), \beta^{n} \rho\left(D, D^{\prime}\right)\right\}, \quad D, D^{\prime} \in \mathcal{C}(E), n=1,2, \cdots
$$

Since $\rho\left(D, D^{\prime}\right)$ is uniformly bounded, letting $n \rightarrow \infty$, we obtain

$$
\rho\left(A_{\alpha}(y), A_{\alpha}(x)\right) \leq \beta d(y, x) \text { for } x, y \in E .
$$

Therefore

$$
\sup _{\alpha \in[0,1]} \rho\left(A_{\alpha}(y), A_{\alpha}(x)\right) \rightarrow 0 \quad(y \rightarrow x) \quad \text { for } x \in E .
$$

Thus the family $\left\{A_{\alpha}(x) \mid x \in E, \alpha \in[0,1]\right\}$ satisfies the conditions (i) - (iii) of Lemma 3. By Lemma 3 , we can define a fuzzy relation $\tilde{c} \in \mathcal{R}(E)$ by

$$
\tilde{c}(x, y):=\sup _{\alpha \in[0,1]}\left\{\alpha \wedge I_{A_{\alpha}(x)}(y)\right\}, \quad x, y \in E .
$$


Then $\tilde{c}_{\alpha}(x)=A_{\alpha}(x)(x \in E, \alpha \in[0,1])$. Since $A_{\alpha}(x)$ is a unique fixed point of $T_{x, \alpha}$,

$$
\lim _{n \rightarrow \infty}\left(\tilde{c}^{n}\right)_{\alpha}(x)=\lim _{n \rightarrow \infty} T_{x, \alpha}^{n}(\{x\})=A_{\alpha}(x)=\tilde{c}_{\alpha}(x), \quad \alpha \in[0,1] .
$$

We get (iii) since the convergence is uniform in $\alpha \in[0,1]$.

Next we show that $\tilde{c}$ is a solution of $(2.1)$. Since $\tilde{c}_{\alpha}(x)=A_{\alpha}(x)$, we note that

$$
\tilde{q}_{\alpha}(x) \cup \tilde{q}_{\alpha}\left(\tilde{c}_{\alpha}(x)\right)=T_{\alpha}\left(\tilde{c}_{\alpha}(x)\right)=\tilde{c}_{\alpha}(x), \quad \alpha \in[0,1] .
$$

If $\alpha>0$, then we have

$$
\left\{y \in E \mid \tilde{q}(x, y) \vee \max _{z \in E}\{\tilde{c}(x, z) \wedge \tilde{q}(z, y)\} \geq \alpha\right\}=\tilde{q}_{\alpha}(x) \cup \tilde{q}_{\alpha}\left(\tilde{c}_{\alpha}(x)\right) .
$$

If $\alpha=0$, then in a similar way to the proof of [2,Lemma 1] we have

$$
\operatorname{cl}\left\{y \in E \mid \max _{x \in E}\left\{\tilde{q}(x, y) \vee \max _{z \in E}\{\tilde{c}(x, z) \wedge \tilde{q}(z, y)\}>0\right\}=\tilde{q}_{0}(x) \cup \tilde{q}_{0}\left(\tilde{c}_{0}(x)\right) .\right.
$$

Therefore

$$
\left\{y \in E \mid \tilde{q}(x, y) \vee \max _{z \in E}\{\tilde{c}(x, z) \wedge \tilde{q}(z, y)\} \geq \alpha\right\}=\tilde{q}_{\alpha}(x) \cup \tilde{q}_{\alpha}\left(\tilde{c}_{\alpha}(x)\right) \quad \text { for } \alpha \in[0,1] .
$$

Together with (2.7), we get

$$
\left\{y \in E \mid \tilde{q}(x, y) \vee \max _{z \in E}\{\tilde{c}(x, z) \wedge \tilde{q}(z, y)\} \geq \alpha\right\}=\tilde{c}_{\alpha}(x) \quad \text { for } \alpha \in[0,1] .
$$

Therefore $\tilde{c}$ satisfies (2.1). We prove the uniqueness of solution of (2.1). Let us denote by $\tilde{c}^{\prime} \in \mathcal{R}(E)$ another solution of (2.1). For $x \in E, \alpha \in[0,1]$, it is shown similarly that $\tilde{c}_{\alpha}^{\prime}(x)=\tilde{q}_{\alpha}(x) \cup \tilde{q}_{\alpha}\left(\tilde{c}_{\alpha}^{\prime}(x)\right)$. That is, $\tilde{c}_{\alpha}^{\prime}(x)$ is a fixed point of $T_{x, \alpha}: \mathcal{C}(E) \mapsto \mathcal{C}(E)$. From the uniqueness of the fixed point, we get $\tilde{c}_{\alpha}^{\prime}(x)=\tilde{c}_{\alpha}(x)$ for $x \in E, \alpha \in[0,1]$. By Lemma $3, \tilde{c}^{\prime}=\tilde{c}$. Thus we get (i).

Finally (ii) is trivial from (2.6), using Lemma 1 and $\tilde{c}_{\alpha}(x)=A_{\alpha}(x)$ for $x \in E, \alpha \in$ $[0,1]$. Thus the proof is completed.

TheOREM 2. It holds that

$$
\tilde{c}(x, y)=\bigvee_{n=1,2, \cdots} \tilde{q}^{n}(x, y), \quad x, y \in E .
$$

Further $\tilde{c}$ is the transitive closure of the fuzzy relation $\tilde{q}$, namely $\tilde{c}$ satisfies (i) - (iii) :

(i) $\tilde{c} \geq \tilde{q}$.

(ii) $\tilde{c}$ has the transitive property :

$$
\tilde{c}(x, y) \geq \sup _{z \in E}\{\tilde{c}(x, z) \wedge \tilde{c}(z, y)\}, \quad x, y \in E .
$$


(iii) If $\tilde{r} \in \mathcal{R}(E)$ satisfies $\tilde{r} \geq \tilde{q}$ and has the transitive property, then $\tilde{r} \geq \tilde{c}$.

Proof. Let $\tilde{r}(x, y):=\bigvee_{n=1,2, \cdots} \tilde{q}^{n}(x, y), x, y \in E$. Then we have $\tilde{r} \geq \bigvee_{n=1,2, \cdots, m} \tilde{q}^{n}=$ $\tilde{c}^{m}$ for $m=1,2, \cdots$. Therefore $\tilde{r}_{\alpha}(x) \supset\left(\tilde{c}^{m}\right)_{\alpha}(x)$ for $x \in E, \alpha \in[0,1], m=1,2, \cdots$. From (2.2) we obtain $\tilde{r}_{\alpha}(x) \supset \tilde{c}_{\alpha}(x)$ for $x \in E, \alpha \in[0,1]$. Thus we get $\tilde{r} \geq \tilde{c}$.

On the other hand, from (2.1), we obtain $\tilde{c} \geq \tilde{q}$ and

$$
\tilde{c}(x, y) \geq \sup _{z \in E}\{\tilde{c}(x, z) \wedge \tilde{q}(z, y)\} \geq \sup _{z \in E}\{\tilde{q}(x, z) \wedge \tilde{q}(z, y)\}=\tilde{q}^{2}(x, y), \quad x, y \in E .
$$

Repeating this argument inductively, we obtain $\tilde{c} \geq \tilde{q}^{n}$ for $n=1,2, \cdots$. Therefore $\tilde{c}(x, y) \geq \tilde{c}^{n}(x, y)$ for $x, y \in E, n=1,2, \cdots$. Thus we get $\tilde{c} \geq \tilde{r}$. Therefore we obtain (2.8).

Next we prove (i) - (iii). (i) is trivial from (2.1). From (2.8), we have

$$
\begin{aligned}
\tilde{c}(x, y) & \geq \bigvee_{n=1,2, \cdots, m ; n^{\prime}=1,2, \cdots, m^{\prime}} \tilde{q}^{n+n^{\prime}}(x, y) \\
& =\sup _{z \in E}\left\{\bigvee_{n=1,2, \cdots, m} \tilde{q}^{n}(x, z) \wedge \bigvee_{n^{\prime}=1,2, \cdots, m^{\prime}} \tilde{q}^{n^{\prime}}(z, y)\right\} \\
& =\sup _{z \in E}\left\{\tilde{c}^{m}(x, z) \wedge \tilde{c}^{m^{\prime}}(z, y)\right\}, \quad x, y \in E .
\end{aligned}
$$

Taking the supremum over $m=1,2, \cdots$ and $m^{\prime}=1,2, \cdots$, we obtain (ii). Finally let $\tilde{r} \in \mathcal{R}(E)$ satisfy $\tilde{r} \geq \tilde{q}$ and have the transitive property. Then

$$
\tilde{r}(x, y) \geq \sup _{z \in E}\{\tilde{r}(x, z) \wedge \tilde{r}(z, y)\} \geq \sup _{z \in E}\{\tilde{q}(x, z) \wedge \tilde{q}(z, y)\}=\tilde{q}^{2}(x, y), \quad x, y \in E .
$$

Repeating this argument inductively, we obtain $\tilde{r} \geq \tilde{q}^{n}$ for $n=1,2, \cdots$. Therefore $\tilde{r}(x, y) \geq \tilde{c}^{n}(x, y)$ for $x, y \in E, n=1,2, \cdots$. Thus we get $\tilde{r} \geq \tilde{c}$. Therefore (iii) holds. The proof is completed.

\section{Numerical example}

Let $E=[-2,2]$ be a space of states. We consider a fuzzy relation (see [2,Figure 1])

$$
\tilde{q}(x, y)=\left\{1-\left|y-\left(\frac{x}{2}+\frac{1}{4}\right)\right|\right\} \vee 0, \quad x, y \in E .
$$

Then we have

$$
\tilde{q}^{n}(x, y)=\left\{1-\left|y-\left(\frac{1}{2^{n}} x+\frac{1}{2}-\frac{1}{2^{n+1}}\right)\right| /\left(2-\frac{1}{2^{n-1}}\right)\right\} \vee 0, \quad x, y \in E, n=1,2, \cdots .
$$

From Theorem 2, we obtain

$$
\tilde{c}(x, y)=\bigvee_{n=1,2, \ldots}\left\{1-\left|y-\left(\frac{1}{2^{n}} x+\frac{1}{2}-\frac{1}{2^{n+1}}\right)\right| /\left(2-\frac{1}{2^{n-1}}\right)\right\} \vee 0, \quad x, y \in E .
$$


Then, (3.3) is the unique solution of Theorem 1 (see Figure 1).

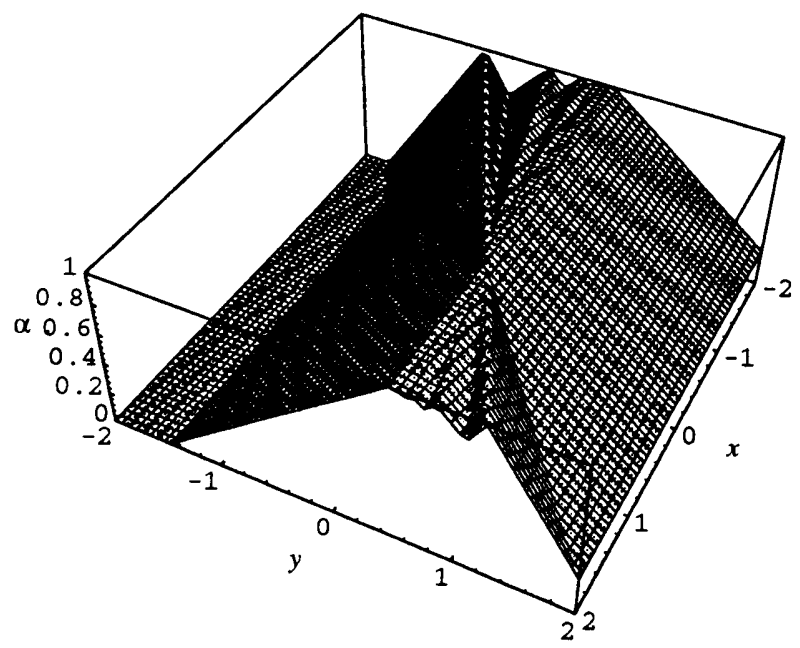

Fig. 1 : The transitive closure $\tilde{c}(x, y)$.

\section{Acknowledgement}

It is a great pleasure for the author to have an opportunity to present a talk in Kitagawa Symposium on Informatics and Statistics. The author also thanks the referee for his helpful advises.

\section{References}

[1] Kuratowski, K.: Topology I. Academic Press, New York, (1966).

[2] Kurano, M., Yasuda, M., Nakagami, J. and Yoshida, Y.: A limit theorem in some dynamic fuzzy systems. Fuzzy Sets and Systems, 51 (1992), 83 - 88.

[3] Nanda, S.: On sequences of fuzzy numbers. Fuzzy Sets and Systems, 33 (1989), 123 $-126$.

[4] Novàk, V.: Fuzzy sets and their applications. Adam Hilder, Bristol-Boston, (1989).

[5] Zimmermann, H.-J.: Fuzzy set theory and its applications. Kluwer-Nijhoff, Boston, (1985).

Received June 15, 1994

Revised September 22, 1994 